\author{
Heather Moulaison Sandy - University of Missouri \\ Andrew Dillon - University of Texas
}

\title{
Mapping the KO Community
}

\begin{abstract}
Knowledge organization $(\mathrm{KO})$ is considered a distinctive disciplinary focus of information science, with strong connections to other intellectual domains such as philosophy, computer science, psychology, sociology, and more. Given its inherent interdisciplinarity, we ask what might a map of the physical, cultural and intellectual geography of the KO community look like? Who is participating in this discipline's scholarly discussion, and from what locations, both geographically and intellectually? Using the unit of authorship in the journal Knowledge Organization, where is the nexus of KO activity, and what patterns of authorship can be identified? What indices can be generated to describe the KO community of researchers as it has evolved? Cultural characteristics were applied as a lens to explore who is and is not participating in the international conversation about KO. World Bank GNI per capita estimates were used to compare relative wealth of countries and Hofstede's Individualism dimension was identified as a way of understanding attributes of countries whose scholars are participating in this dialog. Descriptive statistics were generated through Excel, and data visualizations were rendered through Tableau Public and TagCrowd. The current project offers one method for examining an international and interdisciplinary field of study, but also suggests potential for analyzing other interdisciplinary areas within the larger discipline of information science.
\end{abstract}

\section{Introduction}

Knowledge organization (KO) is sometimes narrowly conceived as a concern of library and information science professionals, but even a quick examination at the affiliations of authors publishing in the field reveals that other intellectual domains such as philosophy, computer science, business, psychology, linguistics, sociology, and more contribute to and find value in its study The subject matter of $\mathrm{KO}$ embraces fundamental questions of what constitutes knowledge as well as practical concerns of how to represent and enable access for others. Accordingly, it can be difficult to characterize and understand the domain of $\mathrm{KO}$ or to position it intellectually both academically and professionally.

Academic journals provide a forum for the exchange of new knowledge in a discipline and serve as a record of the contributions made to a domain or field across time. As such, a scholarly journal serves to validate research, and by extension, helps to shape the legitimacy of a field of enquiry. Long-standing journals in a domain are considered to provide a measure of prestige for authors as well as an identity for a discipline. New areas of enquiry or research involving non-traditional methods often face a challenge gaining a foothold in academia until a suitable peer-reviewed outlet such as an academic journal or high prestige conference accepts the work for publication or presentation.

By virtue of this type of gatekeeping role, academic journals can provide useful indices of the development of a domain and the research participants within it. Consequently, it is possible to use the back issues of a journal as a test base for examining the emergence, duration, and impact of ideas within a field, as well as the productivity of key scholars. Darmani, Dwaikat, and Portilla (2013), for example, analyzed ten years of contributions to the Journal of Creative Innovation and Management to shed light on how the field of innovation management is evolving over time and to determine the geographical make up of scholarship in the domain. By characterizing author geography, publication trends, recurring themes across a decade, they provided evidence of the diminishing occurrence of single-author papers, the recent growth of scholarship from 
emerging economies, and the dominance of leadership as a primary research emphasis. Similarly, Wiid, du Preez, and Wallström (2012) performed an analysis of Marketing Intelligence and Planning to identify major author patterns and content trends in the field of marketing, highlighting the location of key authors and the productivity of regions in generating new scholarship. Such work can prove useful in encouraging a shared perspective and identifying areas of need within a subject or discipline. The present paper represents an attempt at a similar analysis in the domain of $\mathrm{KO}$.

Efforts to Assess the KO Community

Previous studies of $\mathrm{KO}$ have addressed questions relating to the field's geographic reach and intellectual focus. Zhao and Wei (2017), for example, study collaborations among Chinese authors in KO from 1992 through 2016. In examining 1,298 articles with Chinese authors published in Web of Science Core Collection KO journals, they find an increase in collaborations over the period of study, including in international collaborations (from 50\% in1992 to $92.53 \%$ in 2016). Likewise, Smiraglia (2015) investigates the field to evaluate the work being done in the area of domain analysis, a unique area of study covered in KO. Beyond KO, scholars in LIS have studied the international contributions to the Journal of the American Society for Information Science and Technology (JASIST) and in the Journal of Documentation (He and Spink 2002) over a fifty-year period at the time when electronic journals were changing the scholarly communication landscape. Analyzing first author affiliations only, these authors report that international contributions increased over the time of study (19501999) for both journals. The extent to which KO mirrors the broader discipline or represents a distinct area with unique or distinctive scholarly characteristics in its corpus remains an open question.

\section{Metrics to Assess Countries, Comparatively}

Broad estimates of global expenditure on research suggests where scholarly efforts are most actively pursued, and it's perhaps not surprising that in 2017 the US and Europe accounted for over $45 \%$ of annual spending on research and development, with China accounting for a further $22 \%$ (Statista 2019). These proportions correlate with the existence and growth of universities globally, though the US continues to dominate regional presence within top research university rankings. Domain or disciplinary differences, though more difficult to determine, also exist and are likely to reflect national and political emphases on research. Chinese universities, for example, are becoming highly ranked in engineering and computer science but less so on liberal arts, which remain dominated by US and European, particularly British, institutions.

Global rankings and expenditures are somewhat limited measures, and we recognize that scholars can, depending on their circumstances, be mobile, gravitating toward and succeeding at institutions that allow for them to investigate questions of interest using the methods that are most applicable. Further, we must acknowledge that scholarship in different countries varies in its reward and recognition, and political and economic support from the public and private sectors. Given the range and the regional differences in support and emphasis for particular research, it is interesting to consider where $\mathrm{KO}$ scholarship is situated and how it is distributed and enacted globally. 
A number of metrics are available to assess cultural differences, the best-known being those put forth by Hofstede, Hofstede, and Minkov (2005). Their metrics, derived from large-scale and long-term surveys, outline six dimensions of culture, and profile countries and regions based on their scores across these dimensions. As imperfect as these metrics may be, they have become widely used in business and research, and offer a starting point for comparing cultures internationally. In particular, the Individualism vs. Collectivism dimension has the potential to provide insight into the collaborative nature of scholarship and the writing process around the world. We might expect, for example, that cultures differing on this dimension also manifest distinctive publication styles in terms of single-authored or collaborative articles. Further, we might anticipate that $\mathrm{KO}$, with its interrogation of knowledge structures and authority might be impacted by cultural distinctions based on power distance or uncertainty avoidance.

Another metric, put forth by the World Bank, assesses relative wealth of a country's citizens by calculating the gross national income (GNI) of the country on a per-capita basis. Limited by virtue of reducing entire populations to a single measure of income, these numbers might provide a basis for comparison and, in conjunction with Hofstede et al.'s dimensions. offer one other gross index to help us better understand what we might term the cultural climate of scholarship.

\section{Mapping the KO Community Authors}

For more than 40 years, the journal Knowledge Organization has served as a primary venue for research and discourse in the field. As such, the journal contains the richest record of the discipline's content, contributors, and trends and is explored here to provide us with a database of research activities in the field. Using the unit of authorship, we seek to identify what countries appear as a nexus of $\mathrm{KO}$ activity, and what patterns of authorship (and co-authorship) can be found in these data? We wish to characterize the KO community of researchers as it has emerged on empirical grounds to better understand how this area is evolving and how it is positioned intellectually.

To begin to explore these questions along with the cultural and disciplinary factors influencing the domain, this research paper maps the geography of Knowledge Organization authorship. The current project explores a method for analyzing an international and interdisciplinary field of study that we hope might prove useful not just for $\mathrm{KO}$ but for other areas of the information discipline in both standalone and comparative studies.

\section{Method}

To assess the question of authorship by nationality based on institutional affiliation, all scholarly articles published in Knowledge Organization from 2009 to 2018 inclusive were examined. New articles that presented research including research articles and revised conference proceedings were considered scholarly and were retained for analysis. ${ }^{1}$ Editorials, book reviews, and reprints of seminal articles were excluded for

\footnotetext{
${ }^{1}$ For this project, scholarly articles retained included articles labeled "peer reviewed" and research articles that expand on peer-reviewed conference proceedings (usually indicated in the TOC as "Selected Papers from the X Conference" - N.B. these tend to be grouped geographically by ISKO chapter, which affects the mapping of authorship in a way that should be acknowledged. These are nonetheless part of the
} 
now but could be further analyzed later. Using the individual author as the primary unit of analysis, each contributor to the publication of a scholarly article in Knowledge Organization was identified, and his or her name, institution, school, department, or unit if applicable, the country of the institution, and the total number of co-authors on the article were retained in Excel.

Hofstede's Individualism-Collectivism dimension was applied to the data set as a way of understanding relative attributes of countries whose scholars are participating in this dialog. World Bank GNI per capita estimates in US dollars for 2017 were used to compare relative wealth of countries. ${ }^{2}$ Because of our assumptions about the mobile nature of academics and the observation that English has become the lingua franca in scholarly communication, no attempt to understand authors' country of origin, languages spoken, or educational background was made. Descriptive statistics were generated through Excel; more complex data visualizations were rendered through Tableau Public and TagCrowd.

\section{Results and Discussion}

For this project, 362 scholarly articles, with 632 individual statements of authors, were coded for analysis and description. In the first instance, we examined publication rates over time and determined that over the last 10 years there has been almost a doubling of published papers in Knowledge Organization (see figure 1), though this might reflect exceptional years 2016-asnd 2017. Nevertheless, the general trend is positive with increasing number of papers published in Knowledge Organization over time.

scholarly record produced by Knowledge Organization, so excluding them would be a mistake.) Finally, Reviews of Concepts in Knowledge Organization were retained. Editorials, features, brief communications, discussions such as the "Forum: The Philosophy of Classification," "Classification Research," "Research Trajectories," conference reports, "ISKO News," book reviews, introductions to special issues, festschrift articles reviewing the life of honorees, and reprintings of previously published articles were not retained for inclusion.

2 https://data.worldbank.org/indicator/ny.gnp.pcap.pp.cd 
Figure 1. Scholarly articles appearing in Knowledge Organization by year. ${ }^{3}$

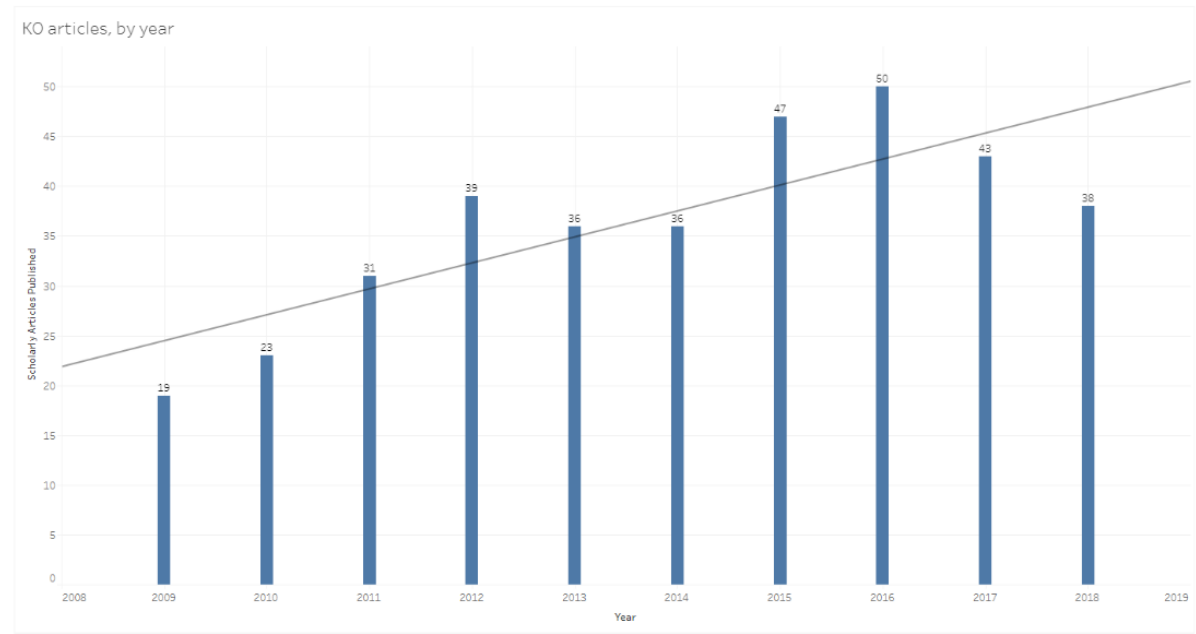

A total of 466 unique authors contributed to the articles, with the majority $(n=384)$ of authors contributing to one article, and a minority $(n=82)$ contributing to two or more articles (see figure 2). What this means for Knowledge Organization as a scholarly venue is not obvious. This might reflect the increasing breadth of new authors publishing in Knowledge Organization or it could be the case of scholars just publishing once here and moving on or not publishing further (in the case of students who publish with professors but then pursue professional careers elsewhere). This is one question that might be usefully pursued over time.

\footnotetext{
${ }^{3}$ Interactive map available online:

https://public.tableau.com/profile/heather8449\#!/vizhome/MappingKOauthorship/KOarticlesbyyear
} 
Figure 2. Number of articles published by each author over the ten-year period.

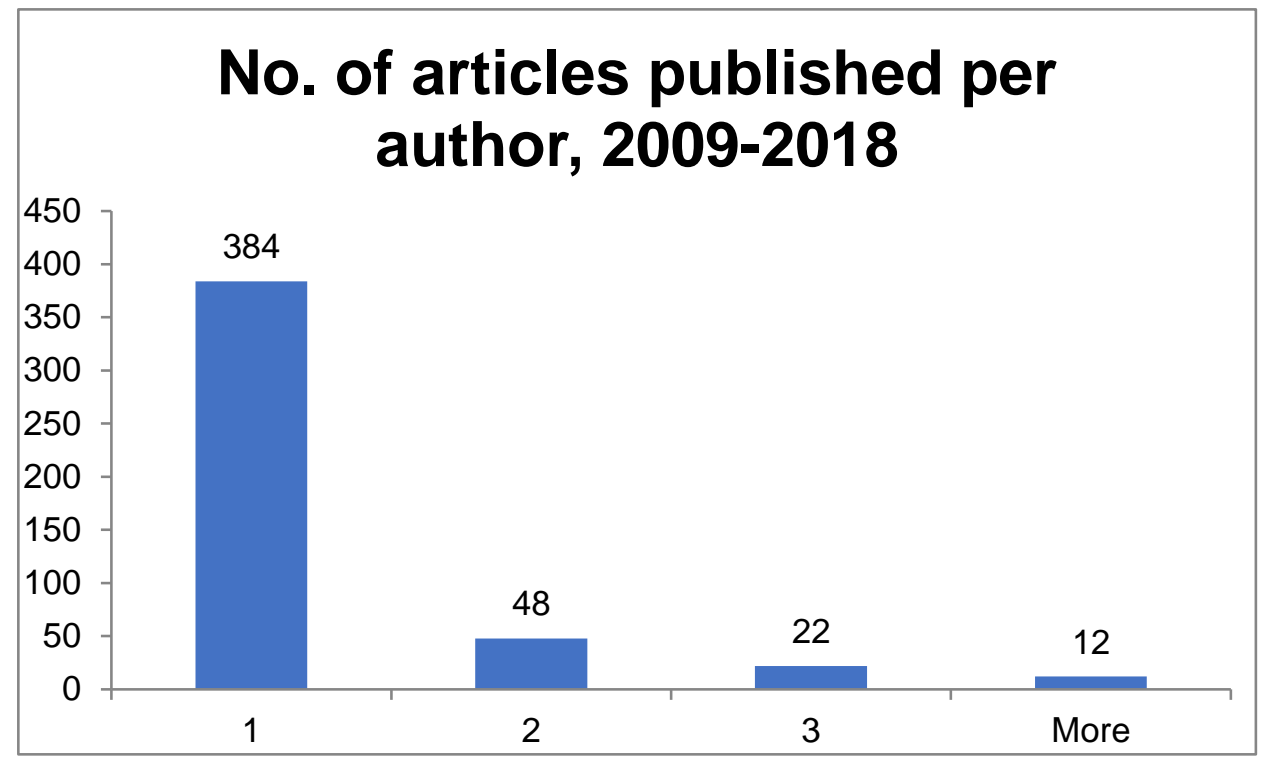

In terms of individual author productivity, twelve authors published four or more scholarly articles over the 10-year period (see Table ). While traditional author impact and productivity measures are not the focus of this work, it is interesting to note that these 12 individuals' contributions represent roughly $24 \%$ of the journal's total output. Without comparative data from other fields it is hard to draw conclusions here but at first glance, this proportion of contributions from a rather small set of scholars might be indicative of an emerging rather than a mature field and is likely of some interest to those involved in promotion and tenure discussions. 
Heather Moulaison Sandy and Andrew Dillon. 2019. Mapping the KO Community. NASKO, Vol. 7. pp. 94 111.

Table 1. Individual authors contributing 4 or more scholarly articles to Knowledge Organization, 2009-2018 and their country and school/department affiliations.

\begin{tabular}{llll}
\hline \multicolumn{1}{c}{ Author } & Country & \multicolumn{1}{c}{ School or department affiliation } & $\begin{array}{c}\text { Articles } \\
\text { contributed }\end{array}$ \\
\hline Birger Hjørland & Denmark & Department of Information Studies & 14 \\
Daniel Martínez-Ávila & Brazil & Department of Information & 12 \\
Claudio Gnoli & Italy & Library & 7 \\
José Augusto Chaves & Brazil & Graduate School of Information Science & 7 \\
Guimarães & USA & School of Information Studies, Knowledge & 7 \\
Richard P. Smiraglia & Canada & Organization Research Group & 7 \\
Elaine Ménard & USA & Information School & 6 \\
Joseph T. Tennis & USA & School of Information Studies & 6 \\
Margaret E. I. Kipp & USA & School of information studies & 6 \\
Melodie J. Fox & Canada & Department of Economics & 6 \\
Rick Szostak. & Brazil & Department of Information Science & 6 \\
Fabio Assis Pinho & Canada & Faculty of Information & 5 \\
Patrick Keilty & & & 4 \\
\hline
\end{tabular}

Authors were affiliated with institutions located in 39 countries. See figure 3 for a breakdown of the number of authors from Algeria to Singapore, by year. This suggests that $\mathrm{KO}$ scholarship is indeed global. As expected, the most productive scholars shown above (table 1) are generally from the countries with the highest representation over time, including the United States, Canada, Brazil, and Denmark. 
Figure 3. Number of Knowledge Organization authors per year, by country. ${ }^{4}$

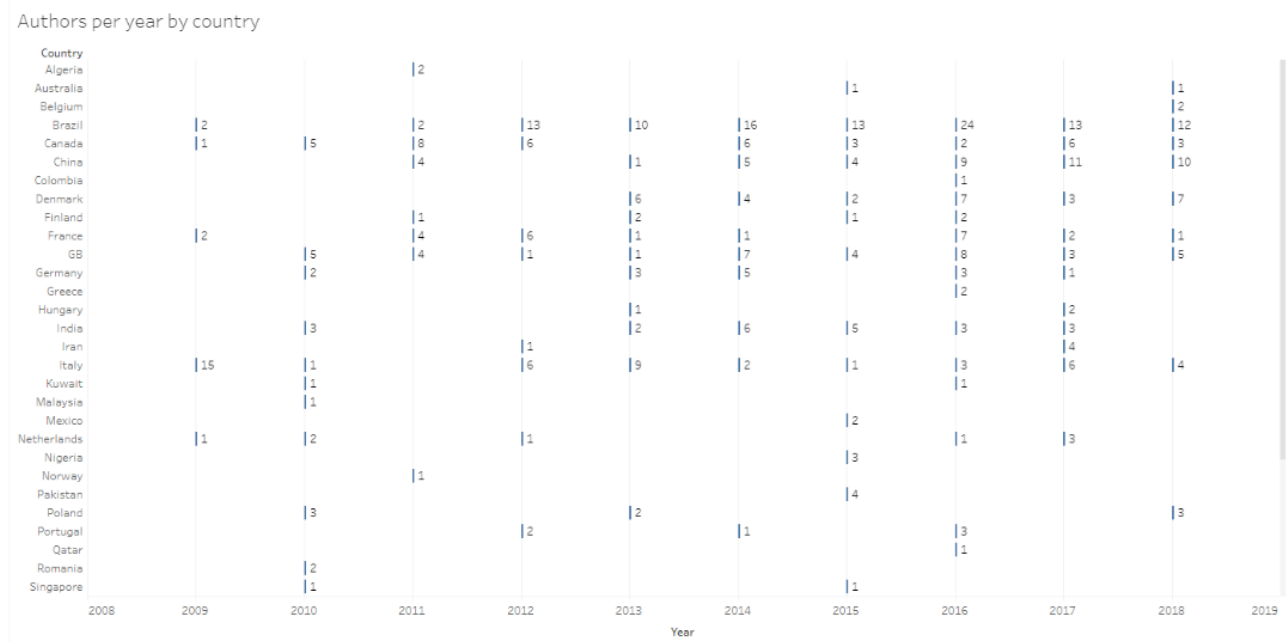

With authors as the unit of analysis, entries for each author responsible for the scholarly articles studied were coded separately. Figure 4 maps the contributions of these authors, by entry for author. Darker blue countries had higher numbers of total author contributions during the 10-year period of study, with the largest number of scholarly article authors coming from the United States $(n=137)$ and Brazil $(n=105)$.

${ }^{4}$ Full visualization can be accessed online: https://public.tableau.com/profile/heather8449\#!/vizhome/MappingKOauthorship/Authorsperyearbycountr y 
Heather Moulaison Sandy and Andrew Dillon. 2019. Mapping the KO Community. NASKO, Vol. 7. pp. 94111.

Figure 4. Average number of authors per article, by country, for 2009-2018 inclusive. ${ }^{5}$

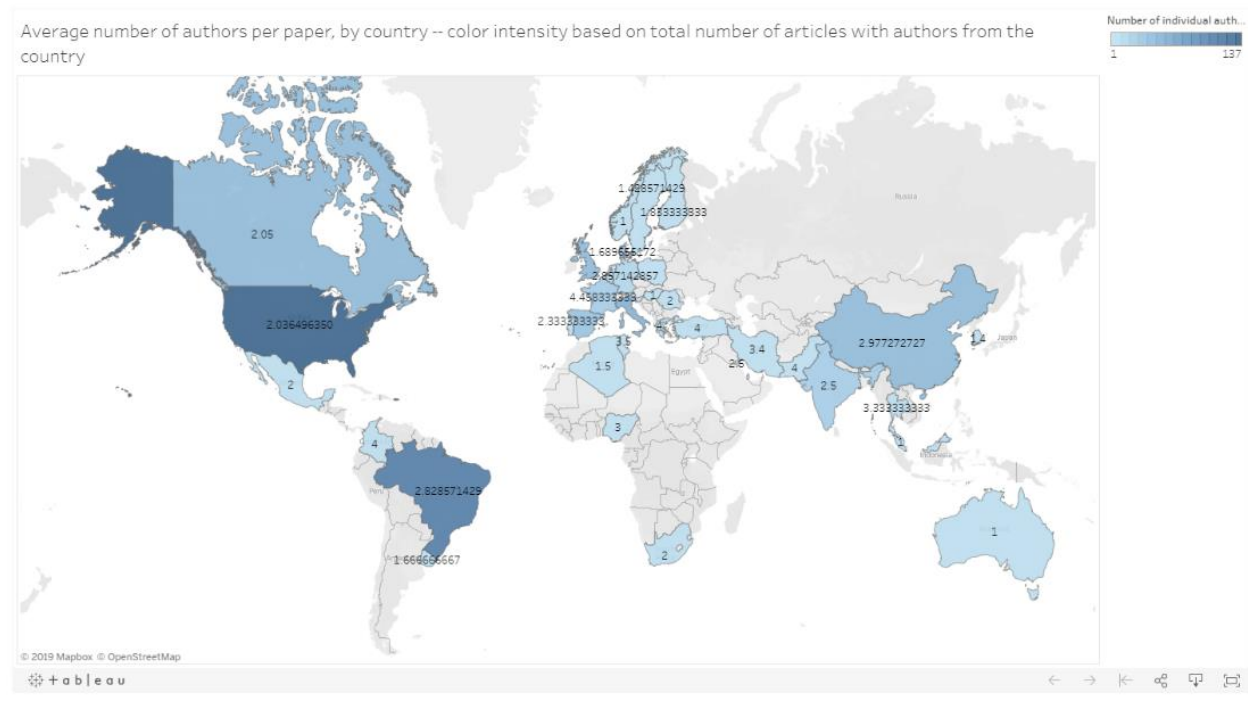

${ }^{5}$ An interactive version of this map is available online: https://public.tableau.com/profile/heather8449\#!/vizhome/MappingKOauthorship/Authorsbycountry 
Heather Moulaison Sandy and Andrew Dillon. 2019. Mapping the KO Community. NASKO, Vol. 7. pp. 94 111.

Figure 5. Distribution of authorship, by country for each year of study. ${ }^{6}$

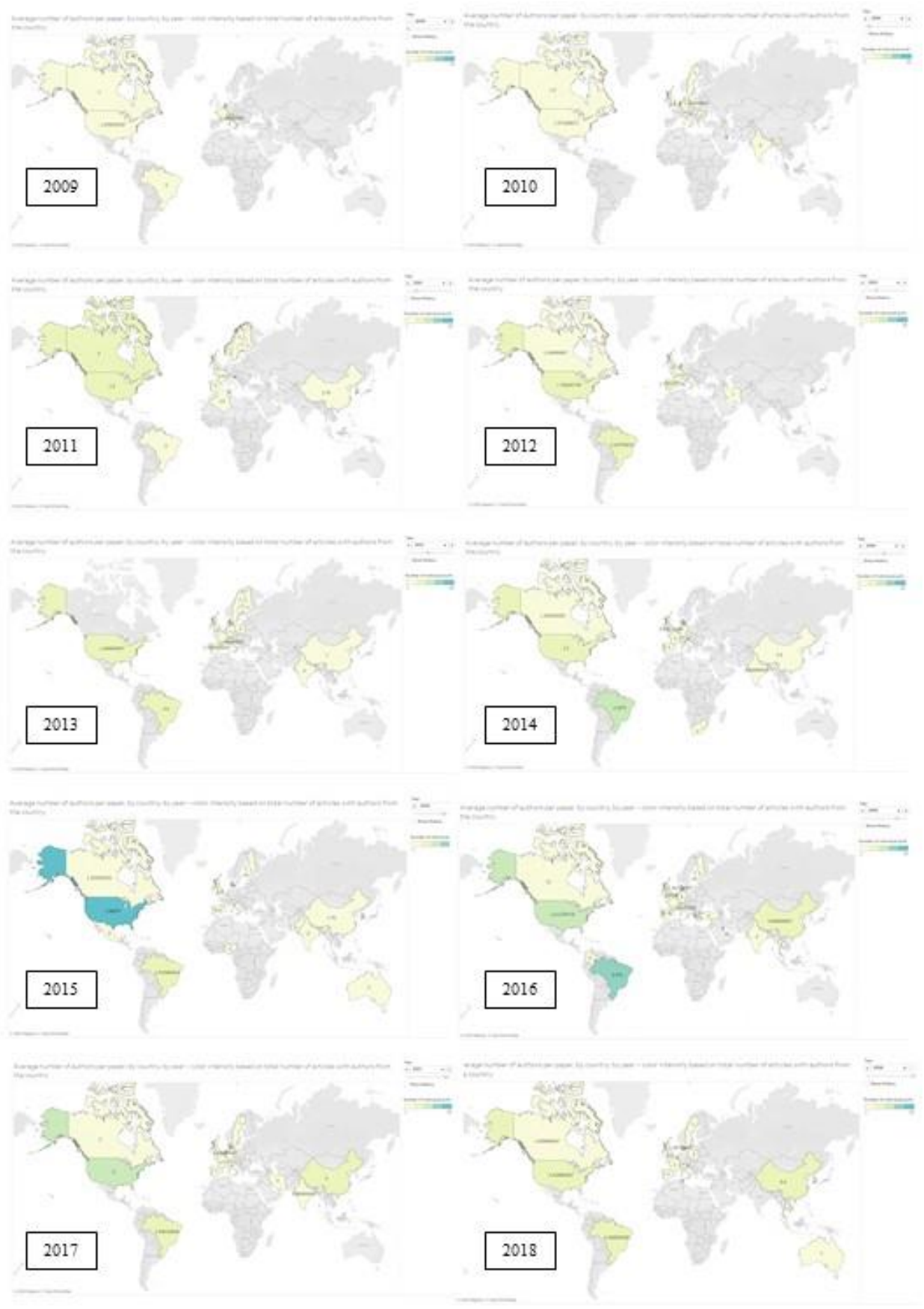


The progression over time of international authorship can be seen in figure 5 (interactive version available online). Also visible is the publication of the revised proceedings of the various biennial ISKO chapter meetings (featured chapters include ISKO France's 2017 conference (2017), ISKO-UK's 2017 conference (2017), ISKOBrazil's 2017 conference (2017), ISKO-Italy's 2017 conference (2017), ISKO-Brazil's 2015 conference (2016), ISKO Spain-Portugal's 2015 conference (2016), ISKOCanada/US's 2015 conference (2015), ISKO-Brazil's 2013 conference (2014), ISKO Spain and Portugal's 2013 conference (2014), German ISKO's 2013 conference (2013),ISKO Italy's 2011 conference (2012), ISKO-France's 2011 conference (2012), and others. The biennial international ISKO conference has also been represented. For example, the ISKO Conference 2016 (2016) was also featured.

Below the national level, we coded authors in terms of institutions, usually universities, and, where provided, with the academic unit such as school, department, college, etc. Taking these names supplied by authors, a broad overview of the disciplinary nature of home units can be generated. Although single instances of affiliations with Departments of Archaeology, for example, are not depicted in the word cloud generated, a sense of the most common departments is available from scanning figure 6. Information is the overarching school/department name, with library, and computer perhaps unsurprisingly next in proportion. Interestingly, communication, management, engineering, economics, business and technology are also well represented, creating at least an initial sense that the view of $\mathrm{KO}$ as naturally interdisciplinary is supported.

\footnotetext{
${ }^{6}$ An interactive version of these maps is available online: https://public.tableau.com/profile/heather8449\#!/vizhome/MappingKOauthorship/Timelapse2009-2018
} 
Figure 6. Word cloud showing alphabetical list of the top 50 of 223 possible words from department or school names, with stop words in a number of languages applied (generated using https://tagcrowd.com/.)

\section{Arts Biblioteconomia Book Business Center Centre chinese ciencias College Communication computer comunicacion Departamento department Economics engineering graduate information Humanities Faculty library management Media philosophy program Psychology Research Resources Royal $S$ Chool science social $_{\text {. }}$ studies Documentacion Documentation Systems Technology Training Unit}

Using Hofstede et al. (2005)'s dimension of Individualism-Collectivism, ${ }^{7}$ authors publishing in Knowledge Organization from countries ranked on this dimension can be compared to the average number of authors on articles. In figure 7, the darker the color of the country, the higher the Individualism index score. As Hofstede et al. remark, "The vast majority of people in our world live in societies in which the interest of the group prevails over the interest of the individual" (p. 90) but it is clear that significant national differences exist. Knowledge Organization has a great deal of interest from authors in what Hofstede et al deem more 'individualist' cultures, including Canada, the United States, Great Britain, and Australia. In fact, Australia, a highly individualistic country, averages one author for paper (N.B., only two papers with an author from Australia were included in the dataset). Farther along the spectrum of the Individualism-Collectivism dimension is China, a more collectivist culture in Hofstede's survey, and indeed Chinese scholars publish papers with an average of over three authors.

\footnotetext{
${ }^{7}$ The spreadsheet of Hofstede dimensions used in this project was downloaded from the following source: https://geerthofstede.com/research-and-vsm/dimension-data-matrix/
} 
Figure 7. Individualism and authorship, by country. ${ }^{8}$

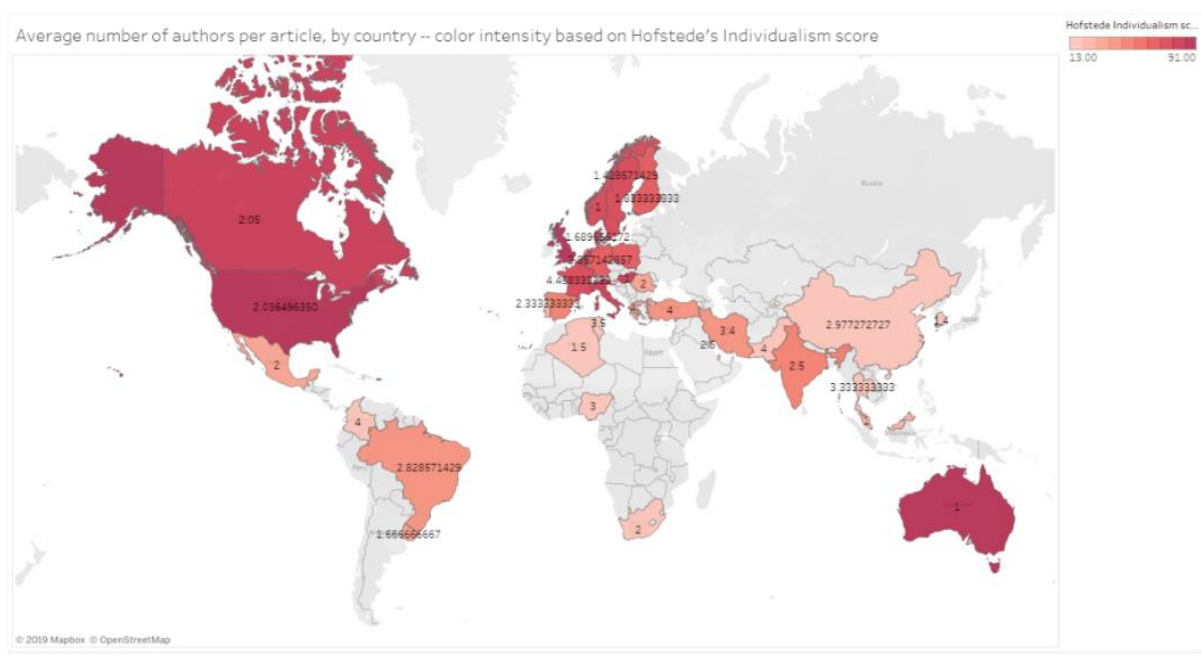

When the average number of authors per article by country is plotted against a country's Individualism-Collectivism dimension score, the trendline reinforces the idea that countries with a higher Individualism score like Canada, Great Britain, the United States, and Australia (averaging between roughly 1 and 2 authors per article) have fewer average authors per article than more collectivist countries such as Colombia and Pakistan, which average 4 authors from their country per article. See figure 8 . The graph, however, is anything but neat, with the bulk of the articles having between 1 and 3 authors regardless of country of origin. The data in figure 8 also represent variations introduced by other cultural dimensions, but nonetheless, even with the caveats we might place on the Hofstede model and the limited data set of Knowledge Organization authorship, these trends present an interesting lens on authorship and co-authorship.

\footnotetext{
${ }^{8}$ An interactive version of this map is available online: https://public.tableau.com/profile/heather8449\#!/vizhome/MappingKOauthorship/HofstedeIndividualism
} 
Figure 8. Hofstede Individualism score, by country, plotted against the average number of authors per country. ${ }^{9}$

Scatterplot IDV x Average No Authors

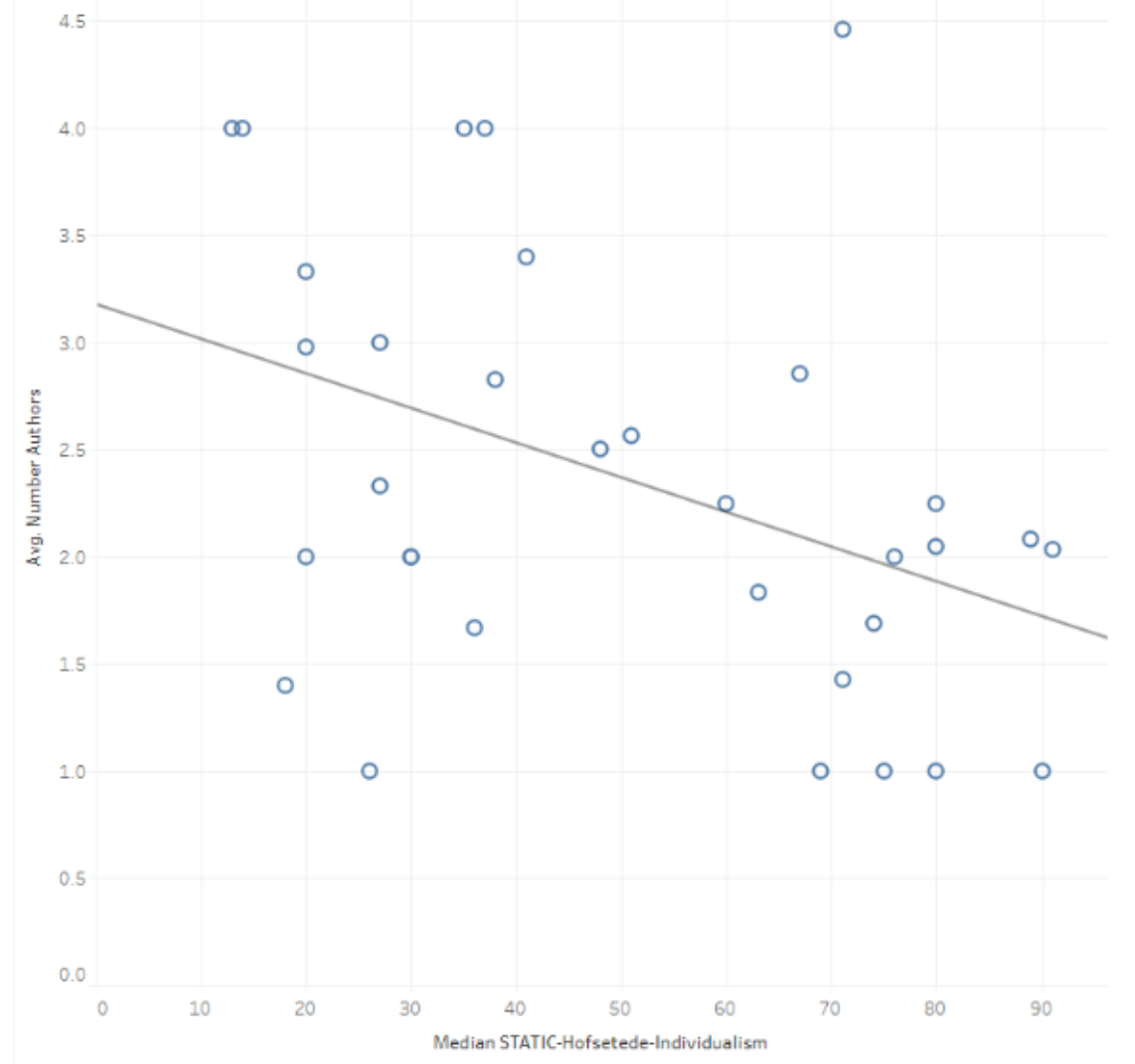

Beyond the rate of single or co-authorship, we might ask if the interests of authors in individualistic and collectivist cultures are similar or different? Based on the author's country of residence, deduplicated lists of the first lines of article titles were used to create word clouds for a group of collectivist countries with Individualism dimension scores between 18-26, all of which are in East Asia (see figure 9). A second word cloud was created based on titles of articles by authors based in the United States (see figure 10). For both, the term "knowledge" was removed given its frequency in all papers. The East Asian titles represent a smaller set of words (113 possible words) and show greater cohesion, with more words displaying with larger font, indicating frequency of use across titles. The presence of 'Chinese', 'Mekong', and 'national' suggest perhaps a concern

\footnotetext{
${ }^{9}$ An interactive version of this figure is available online: https://public.tableau.com/profile/heather8449\#!/vizhome/MAS/ScatterplotIDVxAverageNoAuthors
} 
for local initiatives. Interestingly, the term "organization" does not appear in the East Asian list, which is somewhat surprising given this journal's coverage. In the US titles (a set of 287 possible words) 'organization' is predominant, with 'analysis', 'domain', and 'ethical' the next most common title terms. Again, one should not draw too firm a conclusion from these trends but they suggest some differences in emphasis on KO scholarship across regions and cultures.

Figure 9. Word cloud showing alphabetical list of the top 50 deduplicated article title words, "knowledge" removed, from countries with Individualism indexes 18-26 (i.e.,

Malaysia, China, Thailand, Singapore, and South Korea) $(n=29)$ (generated using https://tagcrowd.com/.).

\section{analysis article chinese construction evaluation evolution greater journal national ontology study Subject support system tags taxonomy}


Figure 10. Word cloud showing alphabetical list of the top 50 deduplicated article title words, "knowledge" removed, from the United States (Individualism score 91) (n=98) (generated using https://tagcrowd.com/.).

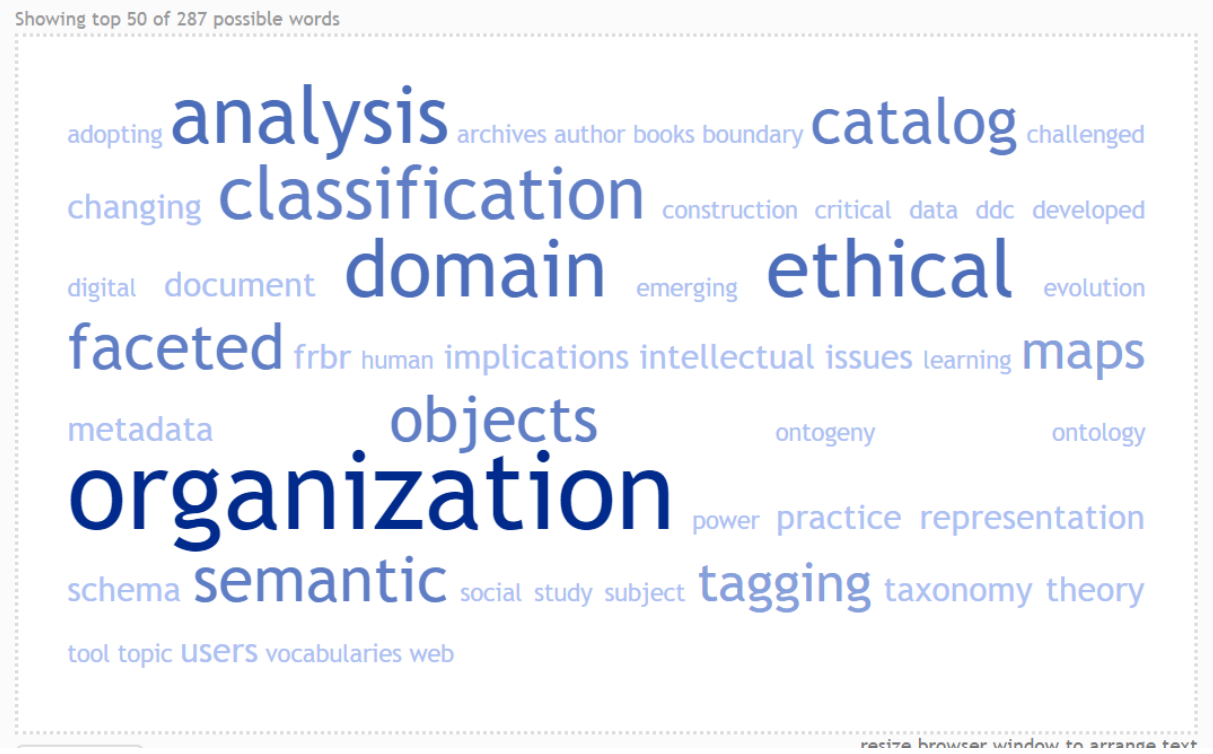

Lastly, in considering the geography of contributions and relative wealth, figure 11 presents a map where countries with larger GNIs are indicated in darker green. Is there a wealth threshold for Knowledge Organization authors? Is $\mathrm{KO}$ the province of richer or wealthier nations? Contributions seem to be somewhat balanced and there is a range of countries on the wealth index participating in $\mathrm{KO}$ but this is clearly a challenge in all disciplines and one that might be usefully explored further in terms of Knowledge Organization's global growth and reach. 
Figure 11. GNI of Knowledge Organization authors' countries, by country. ${ }^{10}$

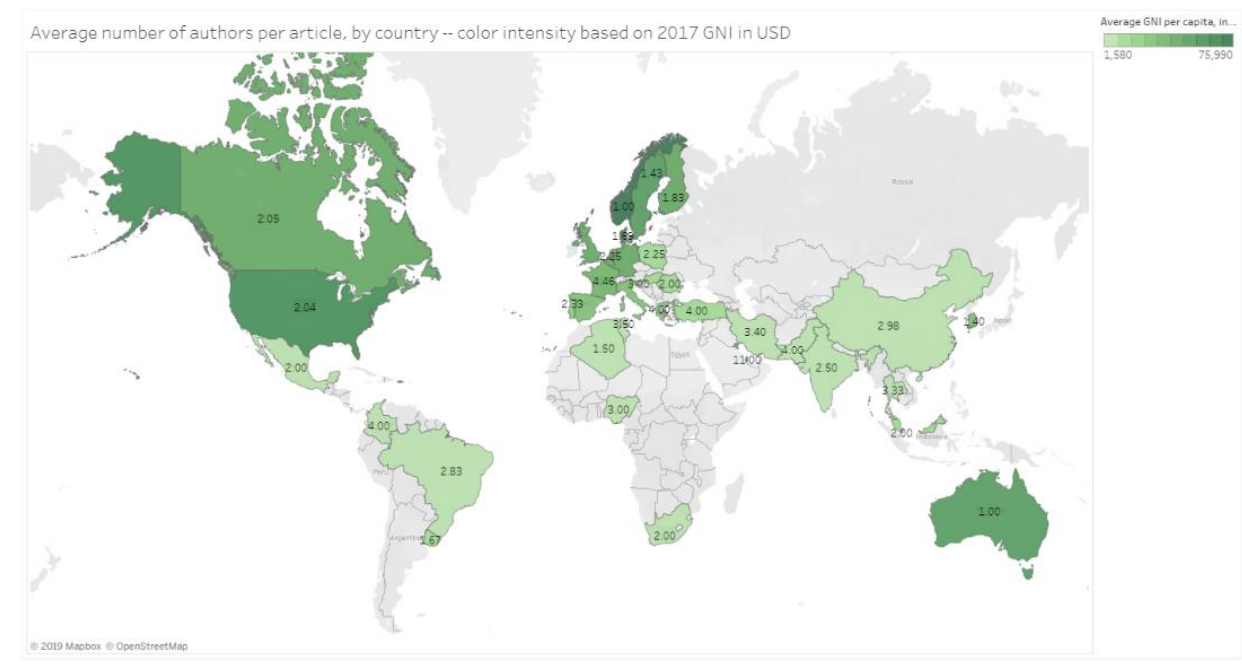

\section{Conclusion}

This research presents a first pass at characterizing the international and interdisciplinary community of scholars publishing in Knowledge Organization. This preliminary analysis suggests four conclusions, with some caveats, as follows:

The publication base is growing. Over the last decade there has been a generally upward growth in the number of articles published in Knowledge Organization, with the article count doubling from 2009-2018.

$\mathrm{KO}$ research is now a global activity, with published papers coming not just from the established scholarly communities in Europe and North America, but from China and other parts of Asia, the Middle East, South America, Africa and Australia, While the numbers in some regions are low, there is reason to be optimistic that $\mathrm{KO}$ is establishing itself internationally as a discipline.

Authorship patterns indicate that co- or group-authorship is routine but the trend in these numbers suggests the broad individualist-collectivist distinction of cultures by Hofstede might help us understand the primary differences among regions on this variable.

Topical analysis suggests that research in KO may also reflect global cultural differences, particularly on the Individualist-Collectivist dimension of Hofstede et al. Our data focused only on two particular regions but is not exhaustive.

There are clearly several limitations to this work. First, we are using data from only one journal. KO is a field practiced outside of English-speaking areas and thus the contributions of non-English language scholars are invisible to this project. Further, this is but a preliminary analysis, using a limited number of measures for a reduced data set of only ten years. While we intend to complete the analysis on the full set of back issues,

\footnotetext{
${ }^{10}$ An interactive version of this map is available online: https://public.tableau.com/profile/heather8449\#!/vizhome/MappingKOauthorship/GNI
} 
fewer research papers were published in the early years. Ideally, we would like to compare $\mathrm{KO}$ with other areas within information science to determine if Knowledge Organization is unique in its pattern of authorship and global activity. Finally, while broad examination of author patterns is interesting, it would be instructive to add a deeper thematic analysis to identify trends in coverage or topics that might indicate how Knowledge Organization is evolving over time as well as across regions. It is important to recognize also that direct conversations with authors, particularly those from different regions, would complement this analysis in terms of author motivations, perceived challenges, and sense of intellectual identity in KO. In sum, we believe there is more work ahead but the early indications are that such analyses of disciplinary records can prove insightful for information scientists.

\section{References}

Darmani, Anna, Nidal Dwaikat, and Andres Ramirez Portilla. 2013. "Twelve Years of Scholarly Research: Content and Trend Analysis of the Journal Creativity and Innovation Management." In 22nd International Conference for Management of Technology IAMOT, April 14th to 18th, 2013, Porto Alegre, Brazil. Retrieved from http://urn.kb.se/resolve?urn=urn:nbn:se:kth:diva-144331

He, Shaoyi, and Amanda Spink. 2002. "A Compairson of Foreign Authorship Distribution in JASIST and the Journal of Documentation." Journal of the American Society of Informatino Science and Technology 53(11): 953-959.

Hofstede, Geert, Gert Jan Hofstede, and Michael Minkov. 2005. Cultures and Organizations: Software of the Mind. New York: McGraw-Hill.

Smiraglia, Richard P. 2015. "Domain Analysis of Domain Analysis for Knowledge Organization: Observations on an Emergent Methodological Cluster." Knowledge Organization 42(8): 602-611.

Statista. 2019. "Distribution of Research and Development (R\&D) Spending Worldwide from 2017 to 2019, by Country/Region." Retrieved from https://www.statista.com/statistics/732224/worldwide-research-anddevelopment-distribution-of-investment/

Wiid, Ria, Rose du Preez, and Åsa Wallström. 2012. "Coming of Age: A 21 Year Analysis of Marketing Intelligence \& Planning from 1990 to 2010." Marketing Intelligence \& Planning 30(1): 4-17.

Zhao, Rongying and Xuqiu Wei. 2017. "Collaboration of Chinese Scholars in International Articles: A Case Study of Knowledge Organization." Knowledge Organization 44(5): 326-334. 